\title{
Efficacy of melatonin for sleep disturbance following traumatic brain injury: a randomised controlled trial
}

\author{
Natalie A. Grima', Shantha M. W. Rajaratnam², Darren Mansfield³ ${ }^{3}$ Tracey L. Sletten², Gershon Spitz² \\ and Jennie L. Ponsford ${ }^{2,4^{*}}$
}

\begin{abstract}
Background: The study aimed to determine the efficacy of melatonin supplementation for sleep disturbances in patients with traumatic brain injury (TBI).

Methods: This is a randomised double-blind placebo-controlled two-period two-treatment (melatonin and placebo) crossover study. Outpatients were recruited from Epworth and Austin Hospitals Melbourne, Australia. They had mild to severe TBI $(n=33)$ reporting sleep disturbances post-injury (mean age 37 years, standard deviation 11 years; 67\% men). They were given prolonged-release melatonin formulation (2 mg; Circadin ${ }^{\oplus}$ ) and placebo capsules for 4 weeks each in a counterbalanced fashion separated by a 48-hour washout period. Treatment was taken nightly 2 hours before bedtime. Serious adverse events and side-effects were monitored.

Results: Melatonin supplementation significantly reduced global Pittsburgh Sleep Quality Index scores relative to placebo, indicating improved sleep quality [melatonin 7.68 vs. placebo 9.47, original score units; difference -1.79; $95 \%$ confidence interval $(\mathrm{Cl}),-2.70$ to $-0.88 ; p \leq 0.0001]$. Melatonin had no effect on sleep onset latency (melatonin 1.37 vs. placebo 1.42 , log units; difference $-0.05 ; 95 \% \mathrm{Cl},-0.14$ to $0.03 ; p=0.23$ ). With respect to the secondary outcomes, melatonin supplementation increased sleep efficiency on actigraphy, and vitality and mental health on the SF-36 v1 questionnaire ( $p \leq 0.05$ for each). Melatonin decreased anxiety on the Hospital Anxiety Depression Scale and fatigue on the Fatigue Severity Scale ( $p \leq 0.05$ for both), but had no significant effect on daytime sleepiness on the Epworth Sleepiness Scale $(p=0.15)$. No serious adverse events were reported.
\end{abstract}

Conclusions: Melatonin supplementation over a 4-week period is effective and safe in improving subjective sleep quality as well as some aspects of objective sleep quality in patients with TBI.

Trial registration: Identifier: 12611000734965; Prospectively registered on 13 July 2011.

Keywords: Sleep, Insomnia, Traumatic brain injury, Acquired brain injury

\section{Background}

Sleep disturbances are commonly reported following traumatic brain injury (TBI), occurring across the spectrum of severity and persisting years following injury $[1,2]$. Insomnia and hypersomnia are among the most prevalent sleep disorders in TBI patients [2,3]. Despite a

\footnotetext{
* Correspondence: jennie.ponsford@monash.edu

${ }^{2}$ School of Psychological Sciences, Monash University, 18 Innovation Walk, Clayton Campus, Wellington Road, Melbourne, Victoria 3800, Australia ${ }^{4}$ Monash-Epworth Rehabilitation Research Centre, Epworth Healthcare, 89 Bridge Road, Richmond 3121, Victoria, Australia

Full list of author information is available at the end of the article
}

high prevalence, sleep disturbances are often overlooked in the TBI population and evidence-based treatments are lacking [3]. Consequently, untreated sleep disturbances following TBI contribute to ongoing cognitive dysfunction [4], poorer rehabilitation outcomes [5], lower productivity [6] and poorer functional status [7].

Although numerous mechanisms may contribute to sleep disturbance in TBI [1], our research demonstrates that TBI is associated with attenuated and delayed melatonin profiles $[8,9]$. Specifically, we observed reduced evening [9] and overnight melatonin [8] production in TBI patients compared to age and sex matched controls, 
with evening melatonin production positively correlated with REM sleep [9]. As endogenous melatonin has sleep-promoting effects and is involved in the circadian control of the sleep-wake cycle, attenuated melatonin profiles may contribute to sleep-related disturbances following TBI. Thus, restoring melatonin with supplementation may help to alleviate sleep disturbances in individuals with TBI. The aim of the current study was to evaluate the effect of melatonin supplementation $(2 \mathrm{mg} / \mathrm{d})$ on sleep quality in patients with TBI reporting sleep disturbances.

\section{Methods}

\section{Trial design}

A randomised placebo-controlled double-blind twoperiod two-treatment crossover phase III clinical trial was conducted. In light of the known heterogeneity between TBI patients and sleep disturbances in this population, the use of a crossover design minimised the influence of confounding covariates, such as injuryrelated characteristics and factors contributing to sleep disturbances, such as mood, anxiety and pain. The study was conducted in accordance with the Declaration of Helsinki and it was approved by Monash University (CF11/1900-2011001061), Epworth HealthCare (52111) and Austin Health (H2013/04950) human research ethics committees. Participants gave informed consent before taking part. The clinical trial was prospectively registered with the Australian New Zealand Clinical Trials Registry (12611000734965) in July 2011.

The study was conducted from August 2011 until November 2016, with the first patient enrolled on 9 April 2012. The 10-week study comprised a 2-week baseline run-in period followed by two treatment periods, during which participants received either melatonin or placebo for 4 weeks, followed by a crossover with the alternative treatment for a further 4 weeks, separated by a 48 -hour washout period. The washout period was chosen to minimise the carry-over of treatment [10-12], and to provide time for medication turnaround and recharging of the Actiwatch.

\section{Participants}

People with acquired brain injury from trauma (TBI) or stroke were eligible to participate in the study. However, as only three stroke patients completed treatment, this manuscript reports only on patients with TBI. Participants were community-dwelling adults, aged 18 to 65 years, who had sustained mild to severe TBI. We recruited participants from Epworth and Austin Hospitals via referrals from physicians and allied health professionals. TBI participants had a history of blunt head trauma with loss of consciousness, and initial Glasgow Coma Scale (GCS) of 3-14 and post-traumatic amnesia
(PTA). All participants had an identifiable sleep complaint corroborated by a Pittsburgh Sleep Quality Index (PSQI) global score $\geq 8$, followed by a confirmed diagnosis of chronic insomnia according to the International Classification of Sleep Disorders, 3rd Edition [13]. A global PSQI of 8 has been shown to have high specificity and sensitivity to a diagnosis of insomnia in patients with TBI [14]. Participants were excluded if they self-reported sleep problems, fatigue or neurological conditions prior to TBI; were pregnant (screened with a blood test); had undertaken trans-meridian travel across more than one time zone or worked night shifts in the preceding three months; were deemed at a high risk of obstructive sleep apnoea by responses on the Berlin Questionnaire; reported consuming non-prescription sleep medication, benzodiazepines or hypnotics in the preceding 6 weeks; or used illicit or psychoactive substances in the previous 12 months. Participants' urine was screened to rule out illicit substance use. Participants were permitted to continue with ongoing rehabilitative treatment and allowable medications (refer to Table 1), provided that the dosage did not change during the study. Participants were not permitted to commence new interventions or medications during the entirety of the trial. To ensure compliance, participants were sent daily text messages asking them to consume their medication and to fill in their sleep diary. They were also contacted weekly to monitor for adverse symptoms. If new medications were needed during the trial, the participant's prescribing physician was contacted and treatment deferred to study completion.

Interested participants were screened for eligibility after providing verbal consent. Their medical history was corroborated from hospital records. Participants satisfying the inclusion criteria were referred to a sleep physician (DM) for clinical examination to rule out respiratory sleep disorders according to the American Academy of Sleep Medicine's international classification of sleep disorders [15]. All participants were deemed in good health, established by physical examination, blood biochemistry, urine toxicology and body mass index (BMI) less than $35 \mathrm{~kg} / \mathrm{m}^{2}$.

\section{Patient involvement}

The rationale for the study was based on self-reports from people with TBI who told us that sleep was a significant problem. This study involved a community-based sample, and no patients, caregivers or laypeople were involved in developing the research question or study design. Neither patients nor laypersons assisted with the conceptualisation of the study or outcome measures, the interpretation of the results or drafting the manuscript. Outcome measures were selected based on previous studies on sleep and TBI in light of their simplicity and high sensitivity and specificity. The burden of intervention on patients was not assessed, though researchers were cognisant of minimising 
Table 1 Baseline characteristics across treatment sequence

\begin{tabular}{|c|c|c|c|}
\hline \multirow[b]{2}{*}{ TBI characteristics } & \multicolumn{2}{|l|}{ Treatment sequence } & \multirow[b]{2}{*}{ Overall $(n=33)$} \\
\hline & Melatonin then placebo $(n=18)$ & Placebo then melatonin $(n=15)$ & \\
\hline Age, mean (SD), years & $35(11)$ & $38(11)$ & $37(11)$ \\
\hline Body mass index, mean (SD), $\mathrm{kg} / \mathrm{m}^{2}$ & $25.5(3.7)$ & $26.1(4.1)$ & $25.7(3.8)$ \\
\hline Males, no. (\%) & $11(61)$ & $11(73)$ & $22(67)$ \\
\hline Paid employment, no. $(\%)^{a}$ & $4(22)$ & $3(20)$ & $7(21)$ \\
\hline Months post-injury, median (IQR Q1-Q3) & $61(28-115)$ & $25(10-72)$ & $46(13-102)$ \\
\hline Lowest GCS, median (IQR Q1-Q3), raw value & $5(3-9)$ & $8(3-13)$ & $6(3-12)$ \\
\hline PTA duration, median (IQR Q1-Q3), days & $21(12-45)$ & $41(27-60)$ & $33(13-47)$ \\
\hline Mild TBI, no. (\%), PTA 0 to $\leq 1$ day & - & $2(13)$ & $2(6)$ \\
\hline Moderate TBI, no. (\%), PTA $>1$ to $\leq 7$ days & $2(11)$ & $1(7)$ & $3(9)$ \\
\hline Severe TBI, no. (\%), PTA > 7 days & $16(89)$ & $12(80)$ & $28(85)$ \\
\hline Patients prescribed medication, no. (\%) & $13(72)$ & $6(40)$ & $19(58)$ \\
\hline Patients using analgesics, no. (\%) & $7(38)$ & $2(13)$ & $9(27)$ \\
\hline Patients using antacids, no. (\%) & $1(6)$ & $1(7)$ & $2(6)$ \\
\hline Patients using antidepressants, no. (\%) & $6(33)$ & $2(13)$ & $8(24)$ \\
\hline Patients using antiepileptics, no. (\%) & $4(22)$ & $3(20)$ & $7(21)$ \\
\hline Patients using multivitamins, no. (\%) & $1(6)$ & $1(7)$ & $2(6)$ \\
\hline Patients using NSAIDs, no. & $1(6)$ & $2(13)$ & $3(9)$ \\
\hline PSQI, global score & $10(3)$ & $12(4)$ & $11(3)$ \\
\hline Sleep latency, minimum, median (IQR Q1-Q3) & $25(15-55)$ & $23(13-52)$ & $24(14-52)$ \\
\hline Sleep efficiency, \%, (IQR Q1-Q3) & $76(82-86)$ & $81(71-83)$ & $81(75-83)$ \\
\hline ESS, score & $7(4)$ & $9(5)$ & $8(5)$ \\
\hline HADS anxiety & $8(4)$ & $8(5)$ & $8(4)$ \\
\hline HADS depression & $11(6)$ & $8(6)$ & $10(6)$ \\
\hline FSS, score, median (IQR Q1-Q3) & $48(39-56)$ & $49(42-59)$ & $49(41-57)$ \\
\hline \multicolumn{4}{|l|}{ SF-36 v1 } \\
\hline Physical functioning (PF), score & $36(16)$ & $42(13)$ & $38(15)$ \\
\hline Role physical (RP), score & $39(14)$ & $38(12)$ & $38(13)$ \\
\hline Role emotional (RE), score & $38(15)$ & $41(11)$ & $39(13)$ \\
\hline Vitality $(V T)$, score & $39(9)$ & $37(10)$ & $38(9)$ \\
\hline Mental health $(\mathrm{MH})$, score & $40(13)$ & $40(9)$ & $40(11)$ \\
\hline Social functioning (SF), score & $38(12)$ & $35(14)$ & $37(13)$ \\
\hline Bodily pain (BP), score & $44(14)$ & $40(12)$ & $42(13)$ \\
\hline General health $(\mathrm{GH})$, score & $42(11)$ & $39(15)$ & $40(13)$ \\
\hline
\end{tabular}

ESS Epworth Sleepiness Scale, FSS Fatigue severity scale, GCS Glasgow coma scale, HADS Hospital Anxiety Depression Scale, IQR inter-quartile range, PSQI Pittsburgh Sleep Quality Index, NSAID nonsteroidal anti-inflammatory drug, PTA Post-traumatic amnesia, SF-36 v1 Short-form health survey, TBI traumatic brain injury

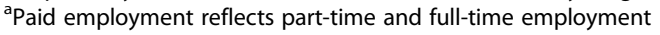

patient burden by limiting the number of questionnaires at follow-up appointments. All follow-up appointments were arranged at the participant's convenience and were limited to less than 1 hour. A summary of results will be disseminated to participants by way of a newsletter, and participants and their families will be encouraged to contact us with questions. Participants will be provided with resources to assist them with obtaining melatonin supplementation.

\section{Intervention}

Participants were randomly allocated to a 4-week melatonin or placebo treatment before crossover to the remaining treatment. Pill containers were labelled with the participant's code and featured instructions for directions as follows: Take one capsule orally at approximately the same time every night, within 2 hours of initiating sleep. To ensure compliance, participants received text messages nightly. Melatonin treatment comprised prolonged-release 
melatonin formula (2 mg, Circadin ${ }^{\circ}$, Sigma Pharmaceuticals Australia). Placebo treatment matched the melatonin treatment for appearance, consisting of mannitol (106 mg), acacia (11 mg) and pure icing sugar (106 mg), compounded by Slade Pharmacy (Melbourne, Australia). Both treatments were encapsulated in identical two-piece gelatin capsules and dispensed in identical 30-capsule containers. This preparation was selected in light of previous studies demonstrating efficacy in individuals with insomnia [16-19], and was the only preparation available in Australia at that time. To monitor for adverse events, at the end of each period, participants were asked if they experienced any of a list of symptoms in the preceding period. If endorsed, symptoms were graded mild, moderate or severe.

\section{Outcome measures}

Age, body mass index, current employment status, TBI injury characteristics, injury date and current medication were recorded at baseline.

\section{Primary end point}

The primary outcomes were sleep quality measured by PSQI global scores, and sleep onset latency measured by wrist actigraphy (Actiwatch-2, Phillips Respironics, Bend, Oregon). The PSQI has 19 items assessing subjectively perceived sleep quality in the previous month, and has been used extensively in patients with TBI. Global scores combine subdomains of sleep duration, sleep disturbance, sleep latency, sleep efficiency, daytime dysfunction, overall sleep quality and medication use. The score range is $0-21$, with higher values indicating poorer sleep quality. The PSQI was completed at the end of baseline and following each treatment phase.

Throughout the study, participants completed a sleep diary retrospectively upon waking to determine time of sleep onset, offset and sleep duration. This was used to corroborate actigraphy sleep variables. To facilitate compliance, participants were sent daily text messages in the morning asking them to complete their sleep diary. Actigraphy data were visually inspected to identify discrepancies between the actigraphy data and the sleep diary. When actigraphy suggested a bedtime or wake time differed by more than 60 minutes from the diary report, the actigraphy was first adjusted by 60 minutes and then by 30 -minute blocks, in combination with activity and light amplitude, as used in a previous study [20]. Actigraphy amendments were undertaken prior to unblinding and verified by a second blinded researcher.

Actigraphy sleep onset latency captures the time elapsed between the start of the rest interval relative to the sleep start time, expressed in minutes. Wrist actigraphy provides a valid measure of sleep-wake behaviour [21]. Participants were instructed to wear the Actiwatch on their non-dominant wrist each day and night, except when bathing or exercising. Actigraphy was collected in 1minute epochs, downloaded and analysed using Actiware ${ }^{\ominus}$ software version 6.0.7 (Philips, Respironics). The sensitivity was set to medium (i.e., 40 activity counts during 1 minute was quantified as awake), as this setting is moderately correlated with polysomnography sleep variables in individuals with insomnia and has been used in the TBI population [20]. Sleep onset latency was averaged during the baseline phase and across each treatment period.

\section{Secondary end points}

Actigraphy sleep efficiency (i.e., total sleep time divided by total duration of the sleep episode, expressed as a percentage) was calculated nightly and averaged over each treatment period. Daytime sleepiness and subjective fatigue were assessed using the Epworth Sleepiness Scale (ESS) and Fatigue Severity Scale (FSS), respectively. The ESS was modified to assess daytime somnolence in the preceding 4 weeks. Self-reported anxiety and depressive symptomology were evaluated with the Hospital Anxiety Depression Scale (HADS). Eight facets of self-reported health-related quality of life were evaluated by the shortform health survey (SF-36 v1). All self-report questionnaires were completed after baseline and following each treatment.

\section{Sample size}

A repeated-measures within-between interaction compromise power analysis was conducted to determine study power (G*Power 3.0.10). Power calculations with an $\alpha=.05$, two repeated measures and 0.7 correlations between repeated measures indicated that 33 participants provided $82 \%$ power to detect a small effect size $(f=.20)$.

\section{Randomisation and blinding}

Participants were randomised to treatment order (melatonin or placebo first) following the 2-week baseline. Treatment randomisation was performed by an independent researcher. Block randomisation was implemented (block size of 4), and the six possible balanced permutations for assignment to the two conditions were assigned an integer from one to six. For each participant, treatment conditions were assigned and sealed in opaque envelopes ( $\mathrm{M}$ for melatonin first and $\mathrm{P}$ for placebo first) and provided to the dispensing pharmacist. Participant codes were allocated sequentially as participants enrolled, and treatments were prepared and allocated by a pharmacist not affiliated with the study. The pharmacist preparing the treatments did not interact with the researchers or participants, and treatments were collected by the research assistants. Participants received one container at the commencement of each treatment period. Participants, physicians and study researchers were blinded to medication allocation. Unblinding of 
treatment occurred after the final participant evaluation. Data were coded by one researcher to indicate which treatment was received first, and those data screened for outliers and missing data. Data were analysed by a separate researcher blinded to treatment allocation.

\section{Statistical analyses}

Intention-to-treat analysis (ITT) was conducted. Treatment efficacy was determined by comparing primary and secondary outcome measures at the end of each treatment. All variables were inspected for normality, with transformations conducted prior to data analysis. Actigraphy sleep efficiency and FSS values were negatively skewed and corrected by applying a reflected square root transformation, with values multiplied by -1 to restore directionality. ESS and actigraphic sleep onset latency were positively skewed and the distributions were normalised by applying logarithmic transformations.

A random-effects mixed-model analysis was used to model each outcome variable as a linear function of treatment (i.e., melatonin or placebo), period (i.e., differences between period 1 and period 2 for placebomelatonin and melatonin-placebo) and sequence (i.e., participants allocated placebo-melatonin vs. participants allocated melatonin-placebo), with participant included as a random variable. Results were considered significant if the two-tailed $p$ value was $<0.05$. Data analysis was performed using STATA (StataCorp. 2015. Stata Statistical Software: Release 14. College Station, TX: Stata Corp LP).

\section{Results}

\section{Participant flow}

Of the 107 TBI individuals referred to the study, 38 consented (Fig. 1) and 35 participants successfully completed baseline and were randomised to treatment. Of the 35 participants randomised, three withdrew during the placebo treatment and no participants withdrew during melatonin treatment. One withdrew after completing the questionnaires after each treatment period and was included in the ITT analysis. The final ITT sample comprised 33 TBI participants. No participant deferred treatment, with all participants receiving treatments as intended.

\section{Baseline data}

Sample demographics are presented in Table 1. All participants were diagnosed with chronic insomnia according to standard criteria [13]. Duration of PTA indicated most participants had severe injuries (88\%). Vehicular accidents were the most frequent mechanism of TBI $(n=23 ; 70 \%)$, followed by pedestrian accidents $(n=6 ; 18 \%)$, falls $(n=3 ; 9 \%)$ and assault $(n=1 ; 3 \%)$.

\section{Numbers analysed and treatment compliance}

All self-report data included 33 participants, except for the HADS $(n=32)$. Actigraphic sleep latency and sleep efficiency reflected data for 31 participants. The actigraphy malfunctioned for one participant and actigraphy data were not collected from one participant who withdrew, but was included in the ITT analysis. Participants wore actigraphy on average for 25 days (Q1$\mathrm{Q} 3=24-28$ days) when receiving melatonin and for 24 days during placebo $(\mathrm{Q} 1-\mathrm{Q} 3=23-28$ days $)$. Paired sample $t$-tests (two-tailed) revealed no differences in the number of days of actigraphy between melatonin and placebo $(t(31)=1.19, p=.244)$.

The majority of participants consumed all 28 of their allocated treatments. Median treatment compliance was 100\% across both treatments (melatonin Q1-Q3 $=97-100 \%$ and placebo Q1-Q3 = 96-100\%). Under both conditions, participants reported taking their assigned treatment as prescribed (melatonin 118 minutes before bed, Q1-Q3 $=106-151$; placebo 128 minutes before bed; Q1-Q3 = 109-170). Circadin was consumed on average at 21:16 hrs (Q1-Q3 = 21:18-21:57), while the placebo treatment was consumed at 20:45 hrs (Q1-Q3 = 20:04-21:45).

\section{Primary outcomes}

Melatonin was associated with a significant and moderate reduction in PSQI global scores, indicating improved sleep quality (Table 2). There was no significant reduction in sleep onset latency with melatonin compared to placebo. The sequence of treatments was not significant across primary and secondary outcome measures (data not shown). Correlational analysis did not reveal any significant association between TBI severity (as measured by PTA or GCS respectively) and effect of treatment for both PSQI or sleep onset latency ( $p>.05$ for all).

\section{Secondary outcomes}

Relative to the placebo, melatonin was associated with a significant but small increase in actigraphic sleep efficiency, indicating a greater maximisation of sleep relative to time in bed (Table 2). Melatonin was also associated with a significant but small decrease in self-reported anxiety symptomatology, with no differences in depression. Melatonin significantly reduced the self-reported impact of fatigue during daily activities as measured by the FSS. With respect to SF-36 v1, melatonin improved self-reported vitality (VT) and mental health $(\mathrm{MH})$. Melatonin was not associated with significant changes on the other six domains of the SF-36 v1, summary scores or ESS.

\section{Safety}

No serious adverse events were reported. Symptoms were more frequently reported during placebo treatment 


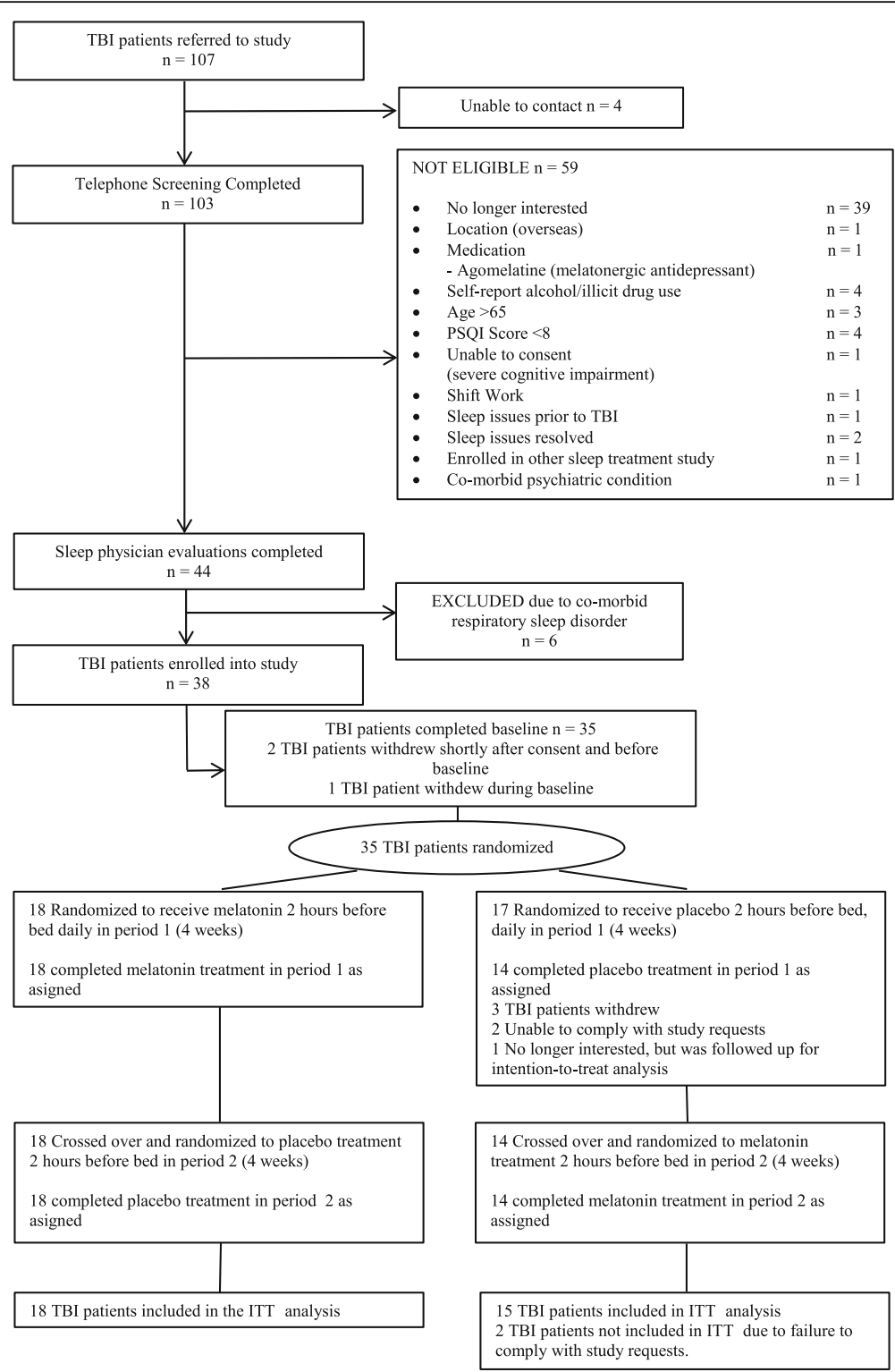

Fig. 1 Overall patient disposition. A total of 35 participants were randomized to treatment. The final ITT sample size comprised of 33 participants. Abbreviations are as follows: ITT intention-to-treat, PSQI Pittsburgh Sleep Quality Index, TBI traumatic brain injury

(Additional file 1: Table S1). The most commonly reported symptoms were neurological, followed by bodily pain, gastrointestinal and dermatologic.

\section{Perceived treatment order}

Participants were asked to guess treatment allocation during the preceding period. The number of participants who guessed correctly was five (28\%) in the melatonin group and eight $(47 \%)$ in the placebo group during period 1 , and seven $(41 \%)$ in the melatonin group and five (28\%) in the placebo group during period 2 .

\section{Discussion}

The aim of the current study was to investigate the efficacy of melatonin in alleviating sleep disturbance in patients with TBI and insomnia. We found that melatonin improved subjective sleep quality and actigraphic sleep efficiency. Melatonin also reduced self-reported anxiety symptomatology and fatigue, whilst improving self-perceived vitality and mental functioning as assessed by the SF-36 v1 health survey. No improvement was observed for daytime sleepiness, depressive symptomatology or the six remaining SF-36 v1 domains. The present results, therefore, suggest that melatonin may be useful in treating sleep disturbances in patients with TBI. 
Table 2 Mixed-model results, including mean estimate under both treatments, the difference of treatments (melatonin minus placebo), effect size and significance level

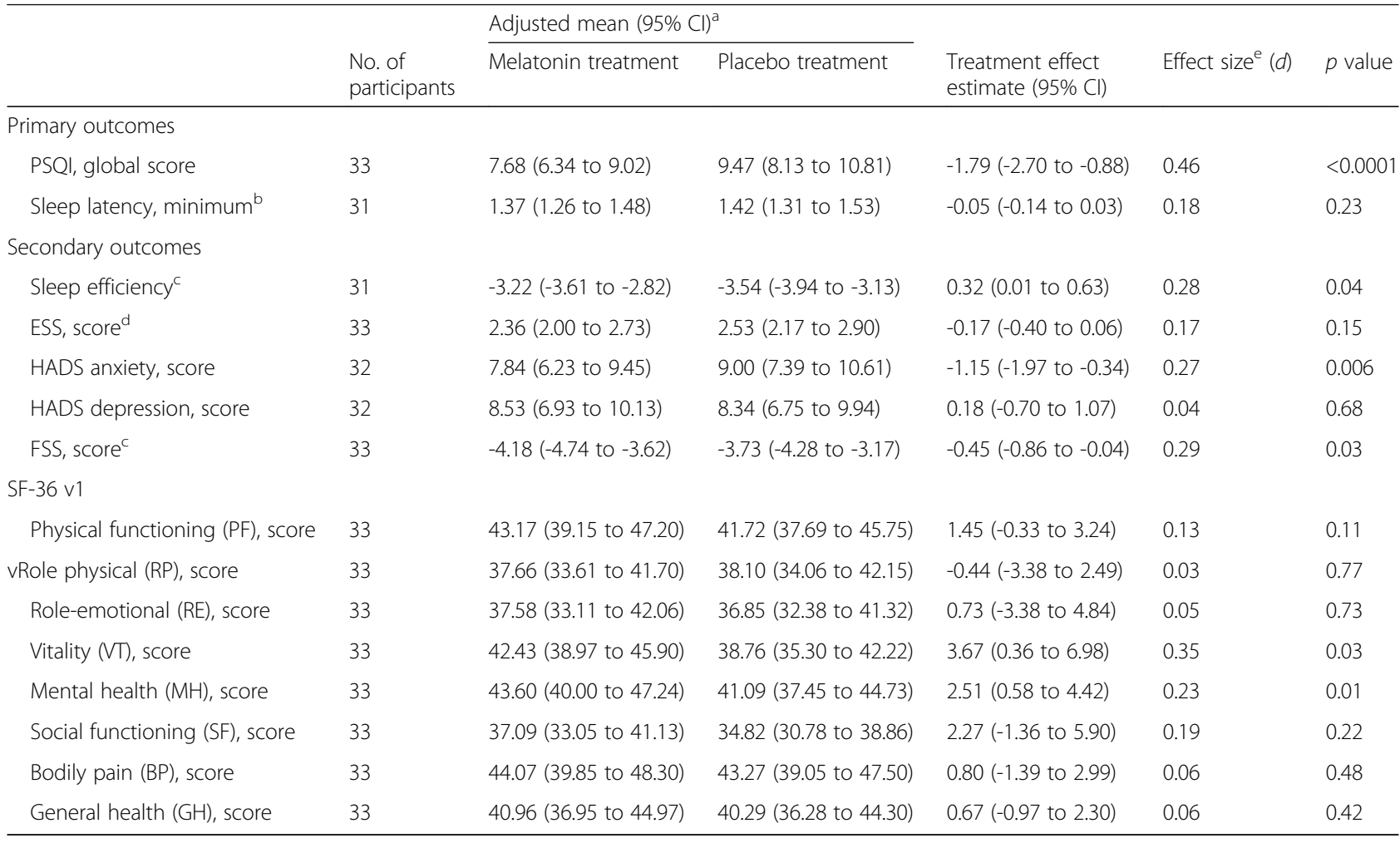

CI confidence interval, ESS Epworth Sleepiness Scale, FSS Fatigue severity scale, HADS Hospital Anxiety Depression Scale, PSQI Pittsburgh Sleep Quality Index ${ }^{a}$ Means adjusted for sequence and period

${ }^{b}$ Variable was log transformed

'Variable was reflected and square root transformed and then multiplied by -1 to restore directionality

dSquare root transformation applied

${ }^{e}$ Cohens $d$ effect size

Our study is limited by the following factors. Firstly, the sample recruited was smaller than intended. Due to the poor recruitment of stroke patients, we cannot determine whether melatonin can improve sleep following a stroke. Secondly, we did not determine endogenous melatonin concentrations and circadian phase in all participants. In a subset of TBI participants $(n=9)$, we demonstrated a $42 \%$ reduction in overnight salivary melatonin production $(d=0.87 ; p=.034)$ and delayed circadian phase $(d=1.23 ; p=.003)$ relative to age and-gender matched controls with similar sleep schedules [8]. As melatonin concentrations and phase were not captured, we are unable to determine the extent to which changes in sleep quality were mitigated by alterations in circadian phase. The aim of the present study was to demonstrate the efficacy of melatonin supplementation in TBI regardless of melatonin production and circadian phase, given that such information is not measured in the clinical setting. The current study was designed to exploit the sleep-promoting effects of melatonin, which have been previously characterised [22], utilising a prolonged-release preparation that mimics the endogenous profile and targets sleep maintenance problems. Therefore, timing of treatment was selected to harness the sleep prompting effects, with time of administration consistent with directions for use as indicated by the manufacturer. Although additional information regarding endogenous melatonin profiles would have been useful, melatonin supplementation was found to be therapeutic. Phenotyping circadian phase (e.g., dim light melatonin onset (DLMO)) would be an important step for future studies by informing the treatment approach by targeting underlying circadian misalignment for maximal benefit. Furthermore, recent discussions regarding the therapeutic outcomes between short-acting vs. long-acting melatonin preparations [23] suggest comparative studies are needed for evidence-based recommendations.

We implemented a 48-hour washout period between consecutive treatments. Although the terminal halflife for melatonin is relatively short (3.5-4 hours) [24], it is possible that melatonin administered in the first treatment period could have induced a circadian phase advance, which could have persisted for up to several days in the following (placebo) treatment period [10-12]. We confirmed that there were no treatment order effects across all outcome measures, and we limited most of the assessment treatment 
outcomes (except sleep diary and actigraphy) to the end of each treatment condition, when circadian phase would have re-established after melatonin treatment had ceased.

The rationale of using a crossover design over a parallel design was to minimise confounding covariates such as TBI characteristics and factors underpinning sleep disturbance, such as depression, anxiety and pain, which were inherently controlled by each participant serving as their own control. The implementation of a crossover design reduced the number of participants required, and it guaranteed all participants received the active treatment.

To our knowledge, only one other study has examined the therapeutic benefit of melatonin on sleep disturbance in TBI. Kemp and colleagues did not find any statistically significant benefits of melatonin on sleep latency, duration, quality or daytime alertness [25]. However, this study was limited to seven males and melatonin was compared to amitriptyline rather than a placebo. With a rigorous study design involving a placebo control and larger sample size, we found melatonin to be efficacious in improving sleep relative to a non-active control.

Previous clinical trials have shown melatonin's sleeppromoting properties in patients with insomnia [16-19] and tetraplegia [26]. Trials that have utilised Circadin $(2 \mathrm{mg})$, the melatonin formulation used in the current study, have shown that this melatonin preparation improves sleep quality [17-19] and sleep efficiency, [16, 27] in people over the age of 55 with insomnia. A similar melatonin formulation has been shown to be effective in improving sleep quality in patients with tetraplegia [26, 28] for whom melatonin profiles are abolished $[29,30]$. Our study extends these findings by showing that a prolonged-release melatonin formation, Circadin, is efficacious in improving sleep quality and sleep efficiency in patients with TBI.

The current study found that melatonin improved subjective sleep quality. Such findings may be attributed to the sleep-promoting properties of melatonin [22]. The temporal association between the nocturnal rise in melatonin and increase in sleep propensity suggests that onset of nocturnal melatonin secretion facilitates the transition from wake to sleep by inhibiting the wake-alerting system $[31,32]$. Melatonin may act as a sleep-promoting agent by attenuating wake-promoting signals from the suprachiasmatic nucleus, primarily via the melatonin MT1 receptor [33]. Melatonin's sleep-promoting properties may also be mediated by the hypothermic response based on the temporal and causal relationship between increases in endogenous melatonin concentrations and subsequent decline in core body temperature [34, 35]. It has also been hypothesised that melatonin may act as a muscle relaxant, [36] and this may explain the apparent impact of melatonin on anxiety symptoms in the present study. The anxiolytic properties of melatonin have been demonstrated in rodents $[37,38]$ and paediatric populations [39]. The improvement in sleep quality also appeared to have a flow-on effect to reduce the subjective impact of fatigue in daily life and improve vitality. Although no clinical global impression was obtained in the current study, a large majority of the outcome measures were based on self-reports by the participants and thus, the current findings are clinically meaningful because changes were perceived by the participants themselves.

The present study required participants to consume treatment approximately 2 hours prior to habitual bedtime and after their evening meal. These instructions are in line with previous studies utilising Circadin $[16-18,40]$ and are based on the pharmacokinetic profile of Circadin $\left(T_{\max }=1.6\right.$ hours) [41]. As a large majority of participants consumed their treatment as prescribed, the translation of the current findings into clinical practice is dependent on appropriately timed melatonin administration.

Various pharmacological treatments exist for the management of sleep disturbance. Hypnotics such as benzodiazepines and non-benzodiazepines are frequently prescribed to patients with TBI experiencing sleep disturbance [42]. Whereas benzodiazepines and non-benzodiazepines are equally effective in treating insomnia in patients with TBI over 7 days [43], the efficacy and safety of long-term use in patients with TBI is unknown. Hypnotics are only indicated for short-term use and prolonged use can result in dependence, especially in vulnerable populations, such as patients with TBI [44]. Conversely, the safety and tolerance profile of melatonin observed in the current study is consistent with previous studies in non-TBI populations [17]. Thus, based on the current findings, melatonin affords TBI patients an alternative treatment to alleviate sleep disturbance with minimal side effects.

In the current study, melatonin had a moderate effect size for improving sleep quality $(d=0.46)$. This magnitude of improvement in sleep quality is double that of amitriptyline (a tricyclic antidepressant) relative to no treatment, when administered to patients with TBI over the same time period $(d=0.21)$ [25]. As melatonin was well tolerated in the current study, with no serious adverse events reported, melatonin offers clinicians an alternative treatment to that of conventional sleeping medications.

While melatonin was found to be effective in alleviating sleep disturbances, it is acknowledged that the mechanisms underpinning sleep disturbances in TBI involve multiple biological and psychological systems, such as alterations in monoaminergic neurons [45, 46] and wake-promoting hypocretin-1 neurons [47], and alterations in melatonin levels $[8,9]$, as well as pain $[48,49]$ and mood disturbance [50]. This suggests that while melatonin supplementation may alleviate sleep 
disturbance in individuals with TBI, melatonin is unlikely to address all sleep problems. Cognitive behavioural therapy for insomnia (CBT-I) has been shown efficacious in TBI populations [51]. Due to the multi-factorial nature of sleep disturbances following TBI, complementing melatonin supplementation with CBT-I may prove beneficial.

\section{Conclusions}

This study provides preliminary evidence for the efficacy of melatonin in alleviating sleep dysfunction in patients with TBI and insomnia. Although melatonin was well tolerated and provides a safe alternative to traditional hypnotic medications in patients with TBI and insomnia, treatment efficacy is unknown over longer durations. Future research should investigate the role of combining melatonin supplementation with other treatments, such as CBT-I and light therapy, to determine improvements in sleep outcomes in TBI beyond that of melatonin alone.

\section{Additional file}

Additional file 1: Table S1. Frequency of symptoms for placebo and melatonin treatments. (DOCX $16 \mathrm{~kb}$ )

\section{Abbreviations}

Cl: Confidence interval; ESS: Epworth Sleepiness Scale; FSS: Fatigue Severity Scale; GCS: Glasgow Coma Scale; HADS: Hospital Anxiety Depression Scale; IQR: Inter-quartile range; ITT: Intention-to-treat; NSAID: Nonsteroidal antiinflammatory drug; PSQI: Pittsburgh Sleep Quality Index; PTA: Post-traumatic amnesia; SF-36 v1: Short-form health survey; TBI: Traumatic brain injury

\section{Acknowledgements}

We thank each and every participant for contributing their time to this study. We would also like to thank Jade Murray for her dedication and assistance with the implementation of the protocol.

\section{Funding}

Funding of the study was supported by an Australian National Health and Medical Research Council project grant (1028733). The funder provided input into study design, but it was not involved in the collection, analysis or interpretation of the data. The funder played no role in the preparation review, writing or approval of the manuscript.

\section{Availability of data and materials}

The data sets generated and/or analysed during the current study are not publicly available as ethics approval was not obtained for releasing the data to anyone other than the primary institution and researchers conducting the study.

\section{Authors' contributions}

NAG monitored data collection for the whole trial, assisted with statistical analysis, cleaned and analysed the data, interpreted the data and drafted the revised paper. She is guarantor. SMWR conceptualised the study, interpreted the data and revised the paper. DM interpreted that data and drafted and revised the paper. TLS assisted with analysing the data and drafted and revised the paper. GS wrote the statistical analysis plan, conducted the statistical analysis, and revised the draft paper. JLP conceptualised the study, interpreted the data, and drafted and revised the paper. All authors had full access to all of the data (including statistical reports and tables) in the study and take responsibility for the integrity of the data and the accuracy of the data analysis. NAG affirms that the manuscript is an honest, accurate and transparent account of the study being reported; that no aspects of the study have been omitted; and that any discrepancies from the study as planned have been explained. All authors read and approved the final manuscript.

\section{Ethics approval and consent to participate}

The study was prospectively approved by the following ethics committees, and participant consent was obtained from each participant before enrolment into the study as per the guidelines outlined by each of the human research committees: Monash University Human Research Committee (CF11/19002011001061), Epworth HealthCare Human Research Committee (52111) and Austin Health Human Research Committee (H2013/04950).

\section{Consent for publication}

Not applicable.

\section{Competing interests}

All authors have completed the International Committee of Medical Journal Editors uniform disclosure form at www.icmje.org/coi_disclosure.pdf. NAG has nothing to disclose. SMWR reports receiving research support outside of the submitted work from Vanda Pharmaceuticals, Phillips Respironics, Teva Pharmaceuticals, Optalert, Philips Lighting, Tyco Healthcare, Compumedics, Rio Tinto and Shell. SMWR also reports that he is the Director of the Sleep Health Foundation as well as the Program Leader of the Cooperative Research Centre for Alertness, Safety and Productivity. SMWR also serves as an advisory board member for Teva Pharmaceuticals (fees paid to Monash University). DM reports receiving grants outside of the submitted work from Fisher Paykel Pty and Rhinomed Pty. TLS reports she serves as a Project Leader in the Cooperative Research Centre for Alertness, Safety and Productivity. GS and JLP have nothing to disclose.

\section{Publisher's Note}

Springer Nature remains neutral with regard to jurisdictional claims in published maps and institutional affiliations.

\section{Author details}

${ }^{1}$ Beth Israel Deaconess Medical Center, 330 Brookline Avenue, Boston 02215, MA, USA. ${ }^{2}$ School of Psychological Sciences, Monash University, 18 Innovation Walk, Clayton Campus, Wellington Road, Melbourne, Victoria 3800, Australia. ${ }^{3}$ Monash Lung and Sleep, Monash Health, 246 Clayton Road, Clayton, Victoria 3800, Australia. ${ }^{4}$ Monash-Epworth Rehabilitation Research Centre, Epworth Healthcare, 89 Bridge Road, Richmond 3121, Victoria, Australia.

Received: 4 August 2017 Accepted: 14 December 2017

Published online: 19 January 2018

\section{References}

1. Ouellet MC, Beaulieu-Bonneau S, Morin CM. Sleep-wake disturbances after traumatic brain injury. Lancet Neurol. 2015;14(7):746-57.

2. Mathias $J \mathrm{~L}$, Alvaro PK. Prevalence of sleep disturbances, disorders, and problems following traumatic brain injury: a meta-analysis. Sleep Med. 2012; 13(7):898-905.

3. Imbach LL, Büchele F, Valko PO, Li T, Maric A, Stover JF, et al. Sleep-wake disorders persist 18 months after traumatic brain injury but remain underrecognized. Neurology. 2016;86(21):1945-9.

4. Mahmood O, Rapport L, Hanks RA, Fichtenberg NL. Neuropsychological performance and sleep disturbance following traumatic brain injury. J Head Trauma Rehabil. 2004:19(5):378-90.

5. Nakase-Richardson R, Sherer M, Barnett SD, Yablon SA, Evans CC, Kretzmer T, et al. Prospective evaluation of the nature, course, and impact of acute sleep abnormality after traumatic brain injury. Arch Phys Med Rehabil. 2013; 94(5):875-82.

6. Beaulieu-Bonneau S, Morin CM. Sleepiness and fatigue following traumatic brain injury. Sleep Med. 2012;13(6):598-605.

7. Fogelberg DJ, Hoffman JM, Dikmen S, Temkin NR, Bell KR. Association of sleep and co-occurring psychological conditions at 1 year after traumatic brain injury. Arch Phys Med Rehabil. 2012;93(8):1313-8.

8. Grima NA, Ponsford JL, St. Hilaire MA, Mansfield DR, Rajaratnam SM Circadian melatonin rhythm following traumatic brain injury. Neurorehabil Neural Repair. 2016;30(10):972-7. 
9. Shekleton JA, Parcell DL, Redman JR, Phipps-Nelson J, Ponsford JL, Rajaratnam SMW. Sleep disturbance and melatonin levels following traumatic brain injury. Neurology. 2010;74(21):1732-8.

10. Sack RL, Brandes RW, Kendall AR, Lewy AJ. Entrainment of free-running circadian rhythms by melatonin in blind people. N Engl J Med. 2000;343(15):1070-7.

11. Hack LM, Lockley SW, Arendt J, Skene DJ. The effects of low-dose 0.5-mg melatonin on the free-running circadian rhythms of blind subjects. J Biol Rhythms. 2003;18(5):420-9.

12. Lockley SW, Skene DJ, James K, Thapan K, Wright J, Arendt J. Melatonin administration can entrain the free-running circadian system of blind subjects. J Endocrinol. 2000;164(1):R1-6.

13. American Academy of Sleep Medicine. International classification of sleep disorders. 3rd ed. Darien: American Academy of Sleep Medicine; 2014.

14. Fictenberg NL, Putnam SH, Mann NR, Zafonte RD, Millard AE. Insomnia screening in postacute traumatic brain injury: utility and validity of the Pittsburgh Sleep Quality Index. Am J Phys Med Rehabil. 2001;80(5):339-45.

15. American Academy of Sleep Medicine. International classification of sleep disorders, revised: diagnostic and coding manual. Chicago: American Academy of Sleep Medicine; 2001.

16. Garkinkel D, Laudon M, Nof D, Zisapel N. Improvement of sleep quality in elderly people by controlled-release melatonin. Lancet. 1995;346(8974):541-4.

17. Wade AG, Ford I, Crawford G, McConnachie A, Nir T, Laudon M, Zisapel N. Nightly treatment of primary insomnia with prolonged release melatonin for 6 months: a randomized placebo controlled trial on age and endogenous melatonin as predictors of efficacy and safety. BioMed Central Med. 2010;8.

18. Wade AG, Ford I, Crawford G, McMahon AD, Nir T, Laudon M, et al. Efficacy of prolonged release melatonin in insomnia patients aged 55-80 years: quality of sleep and next-day alertness outcomes. Curr Med Res Opin. 2007; 23:2597-605.

19. Lemoine $P$, Nir T, Laudon M, Zisapel N. Prolonged-release melatonin improves sleep quality and morning alertness in insomnia patients aged 55 years and older and has no withdrawal effects. J Sleep Res. 2007;16(4): 372-80

20. Sinclair KL, Ponsford JL, Rajaratnam SMW. Actigraphic assessment of sleep disturbances following traumatic brain injury. Behav Sleep Med. 2012

21. Ancoli-Israel S, Cole R, Alessi C, Chambers M, Moorcroft W, Pollak CP. The role of actigraphy in the study of sleep and circadian rhythms. Sleep. 2003, 26(3):342-92.

22. Rajaratnam SMW, Middleton B, Stone BM, Arendt J, Dijk DJ. Melatonin advances the circadian timing of EEG sleep and directly facilitates sleep without altering its duration in extended sleep opportunities in humans. J Physiol. 2004;561(1):339-51.

23. Bruni O, Alonso-Alconada D, Besag F, Biran V, Braam W, Cortese S, et al. Immediate and prolonged-release melatonin in children with neurodevelopmental disabilities. Author reply to Prof. Zisapel. Eur J Paediatr Neurol. 2017:21(2):420-1.

24. Department of Health and Ageing, Therapeutic Goods Administration. Australian public assessment report for melatonin: circadin. Canberra: Australian Government; 2011. Online access: https://www.tga.gov.au/sites/ default/files/auspar-circadin-110118.pdf.

25. Kemp S, Biswas R, Neumann V, Coughlan A. The value of melatonin for sleep disorders occurring post-head injury: a pilot RCT. Brain Inj. 2004;18(9):911-19.

26. Spong J, Kennedy GA, Tseng J, Brown DJ, Armstrong S, Berlowitz DJ. Sleep disruption in tetraplegia: a randomised, double-blind, placebo-controlled crossover trial of 3 mg melatonin. Spinal Cord. 2014;52(8):629-34.

27. Haimov I, Lavie P, Laudon M, Herer P, Vigder C, Zisapel I. Melatonin replacement therapy of elderly insomniacs. Sleep. 1995;18(7):598-603.

28. Spong J, Kennedy GA, Brown Jr RD, Armstrong BG, Berlowitz D. Melatonin supplementation in patients with complete tetraplegia and poor sleep. Sleep Disord. 2013;2013(128197):1-8.

29. Scheer FAJL, Zeitzer JM, Ayas NT, Brown R, Czeisler CA, Shea SA. Reduced sleep efficiency in cervical spinal cord injury; association with abolished night time melatonin secretion. Spinal Cord. 2006;44(2):78-81.

30. Zeitzer JM, Ayas NT, Shea SA, Brown R, Czeisler CA. Absence of detectable melatonin and preservation of cortisol and thyrotropin rhythms in tetraplegia. J Clin Endocrinol Metab. 2000;85(6):2189-96.

31. Shochat T, Luboshitzky R, Lavie P. Nocturnal melatonin onset is phase locked to the primary sleep gate. Am J Physiol. 1997;273(1 Pt 2):R364-70.

32. Lavie P. Melatonin: role in gating nocturnal rise in sleep propensity. J Biol Rhythm. 1997;12(6):657-65.
33. Liu C, Weaver DR, Jin X, Shearman LP, Pieschl RL, Gribkoff VK, et al. Molecular dissection of two distinct actions of melatonin on the suprachiasmatic circadian clock. Neuron. 1997;19(1):91-102.

34. Rajaratnam SMW, Cohen DA, Rogers NL. Melatonin and melatonin analogues. Sleep Med Clin. 2009;4(2):179-93.

35. Cagnacci A, Elliott JA, Yen SSC. Melatonin: a major regulator of the circadian rhythm of core temperature in humans. J Clin Endocrinol Metab. 1992;75:447-52.

36. Rogers NL, Dinges DF, Kennaway DJ, Dawson D. Potential action of melatonin in insomnia. Sleep. 2003;26(8):1058-9.

37. Pierrefiche $\mathrm{G}$, Zerbib R, Laborit $\mathrm{H}$. Anxiolytic activity of melatonin in mice: involvement of benzodiazepine receptors. Res Commun Chem Pathol Pharmacol. 1993;82(2):131-42.

38. Golombek DA, Martini M, Cardinali DP. Melatonin as an anxiolytic in rats: time dependence and interaction with the central GABAergic system. Eur J Pharmacol. 1993;237(2-3):231-6.

39. Marseglia L, D'Angelo G, Manti S, Aversa S, Arrigo T, Reiter RJ, et al. Analgesic, anxiolytic and anaesthetic effects of melatonin: new potential uses in pediatrics. Int J Mol Sci. 2015;16(1):1209-20.

40. Wade AG, Crawford G, Ford I, McConnachie A, Nir T, Laudon M, et al. Prolonged release melatonin in the treatment of primary insomnia: evaluation of the age cut-off for short- and long-term response. Curr Med Res Opin. 2011;27(1):87-98.

41. Department of Health and Ageing, Therapeutic goods administration. Australian Public Assessment Report for Melatonin. In: Australian Government. 2009. p. 42-53.

42. Larson EB, Zollman FS. The effect of sleep medications on cognitive recovery from traumatic brain injury. J Head Trauma Rehabil. 2010;25(1):61-7.

43. Li Pi Shan RS, Ashworth NL. Comparison of lorazepam and zopiclone for insomnia in patients with stroke and brain injury: a randomized, crossover, double-blinded trial. Am J Phys Med Rehabil. 2004;83(6):421-7.

44. Cunnington D, Junge MF, Fernando AT. Insomnia: prevalence, consequences and effective treatment. Med J Aust. 2013;199(8):S36-40.

45. Valko PO, Gavrilov V, Yamamoto M, Noain D, Reddy H, Haybaeck J, et al. Damage to arousal-promoting brainstem neurons with traumatic brain injury. Sleep. 2016;39(6):1249-52.

46. Valko PO, Gavrilov YV, Yamamoto M, Finn K, Reddy H, Haybaeck J, et al. Damage to histaminergic tuberomammillary neurons and other hypothalamic neurons with traumatic brain injury. Ann Neurol. 2014;77(1):177-82.

47. Baumann CR. Loss of hypocretin (orexin) neurons with traumatic brain injury. Ann Neurol. 2009;66(4):555-9.

48. Lavigne $\mathrm{G}$, Khoury S, Chauny JM, Desautels A. Pain and sleep in postconcussion/mild traumatic brain injury. Pain. 2015;156 Suppl 1:S75-85

49. Khoury S, Chouchou F, Amzica F, Giguère JF, Denis R, Rouleau GA, et al. Rapid EEG activity during sleep dominates in mild traumatic brain injury patients with acute pain. J Neurotrauma. 2013:30(8):633-41.

50. Ponsford $J L$, Parcell DL, Sinclair KL, Roper M, Rajaratnam SMW. Changes in sleep patterns following traumatic brain injury: a controlled study. Neurorehabil Neural Repair. 2013;27(7):613-21.

51. Nguyen S, McKay A, Wong D, Rajaratnam SMW, Spitz G, Williams G, et al. Cognitive behavior therapy to treat sleep disturbance and fatigue after traumatic brain injury: a pilot randomized controlled trial. Arch Phys Med Rehabil. 2017:98(8):1508-1517.e1502.

\section{Submit your next manuscript to BioMed Central and we will help you at every step:}

- We accept pre-submission inquiries

- Our selector tool helps you to find the most relevant journal

- We provide round the clock customer support

- Convenient online submission

- Thorough peer review

- Inclusion in PubMed and all major indexing services

- Maximum visibility for your research

Submit your manuscript at www.biomedcentral.com/submit 